\title{
Potential role of the cAMP/PKA/CREB signalling pathway in hypoxic preconditioning and effect on propofol-induced neurotoxicity in the hippocampus of neonatal rats
}

\author{
RUICONG GUAN, JING LV, FEI XIAO, YOUBING TU, YUBO XIE and LI LI \\ Department of Anaesthesiology, The First Affiliated Hospital of Guangxi Medical University, \\ Nanning, Guangxi Zhuang Autonomous Region 530021, P.R. China
}

Received August 23, 2018; Accepted May 29, 2019

DOI: $10.3892 / \mathrm{mmr} .2019 .10397$

\begin{abstract}
Hypoxic preconditioning (HPC) is neuroprotective against ischaemic brain injury; however, the roles of potential anti-apoptotic signals in this process have not been assessed. To elucidate the molecular mechanisms involved in HPC-induced neuroprotection, the effects of HPC on the cyclic adenosine monophosphate (cAMP)/protein kinase A (PKA)/cAMP response element-binding protein (CREB) signalling pathway and apoptosis in Sprague-Dawley pups (postnatal day 7) treated with propofol were investigated. Western blot and histological analyses demonstrated that HPC exerts multiple effects on the hippocampus, including the upregulation of cAMP and phosphorylation of CREB. These effects were partially blocked by intracerebroventricular injection of the protein kinase antagonist H89 $(5 \mu \mathrm{mol} / 5 \mu \mathrm{l})$. Notably, the level of cleaved caspase-3 was significantly downregulated by treatment with the cAMP agonist Sp-cAMP $(20 \mathrm{nmol} / 5 \mu \mathrm{l})$. The results indicate that propofol increased the level of cleaved caspase- 3 and Bax by suppressing the activity of cAMP-dependent proteins and $\mathrm{Bcl}-2$; thus, HPC prevents propofol from triggering apoptosis via the cAMP/PKA/CREB signalling pathway.
\end{abstract}

\section{Introduction}

In the last several years, the importance of propofol as a short-acting anaesthetic agent has begun to be recognized in animal models $(1,2)$. Propofol affects $\mathrm{GABA}_{\mathrm{A}}$ transmission and decreases glutamate transmission (3). These findings have raised questions about how extensively propofol is used and

Correspondence to: Professor Yubo Xie or Dr Li Li, Department of Anaesthesiology, The First Affiliated Hospital of Guangxi Medical University, 6 Shyanghyong Road, Nanning, Guangxi Zhuang Autonomous Region 530021, P.R. China

E-mail: xybdoctor@163.com

E-mail: 3196274@qq.com

Key words: hypoxic preconditioning, propofol, hippocampus, apoptosis what other irreversible effects it exerts on the central nervous system (4-6). Exposure to a subanaesthetic dose of propofol was demonstrated to alter long non-coding RNA profiles in the immature mouse hippocampus (7) and cause disorders in hippocampal circuits resulting in several diseases, including Alzheimer's disease and Parkinson's disease (8), while exposure to a high propofol dose inhibited long-term potentiation in the CA1 area of the adult hippocampus. A $100 \mathrm{mg} / \mathrm{kg}$ dose of propofol induces the expression of apoptotic proteins, including B-cell lymphoma 2-associated X and caspase-3, in Sprague-Dawley pups (postnatal day 7), followed by adverse effects, such as learning and memory impairment (9-12). Therefore, it is hypothesized that neonatal rats have increased sensitivity and are more vulnerable to a $100 \mathrm{mg} / \mathrm{kg}$ dose of propofol than adult rats.

Hypoxic preconditioning (HPC) is the exposure of an organ to a moderate hypoxic stimulus prior to injury (13). Calcium overload (14) and overproduction of reactive oxygen species (15) have been identified by the detection of electrical simulation and neuronal depolarization during cellular processes in the rat hippocampus.

HPC has long been recognized to induce neuroprotection and neuroplasticity in bone marrow stromal cells $(16,17)$; however, the anti-apoptotic signals that mediate these processes remain unclear. To address this issue, immature male Sprague-Dawley rats were exposed to HPC and propofol, either alone or in the relevant combinations. It was hypothesized that HPC increases the concentration of cyclic adenosine monophosphate (cAMP) via direct phosphorylation of effector proteins and regulation of transcriptional activators or the corresponding gene transcription. cAMP response element-binding protein (CREB) is required for neuronal growth within hippocampal tissues. The role of the $\mathrm{cAMP} / \mathrm{CREB}$ signalling pathway in intrinsic apoptosis is illustrated in Fig. 1.

\section{Materials and methods}

Rat HPC model. All animal procedures were conducted with the approval of the Animal Care and Use Committee of Guangxi Medical University (Nanning, China). Seven-day-old (P7) male Sprague-Dawley pups (average body weight, 10-15 g, $\mathrm{n}=70$ ) were identified and numbered using picric acid, which 


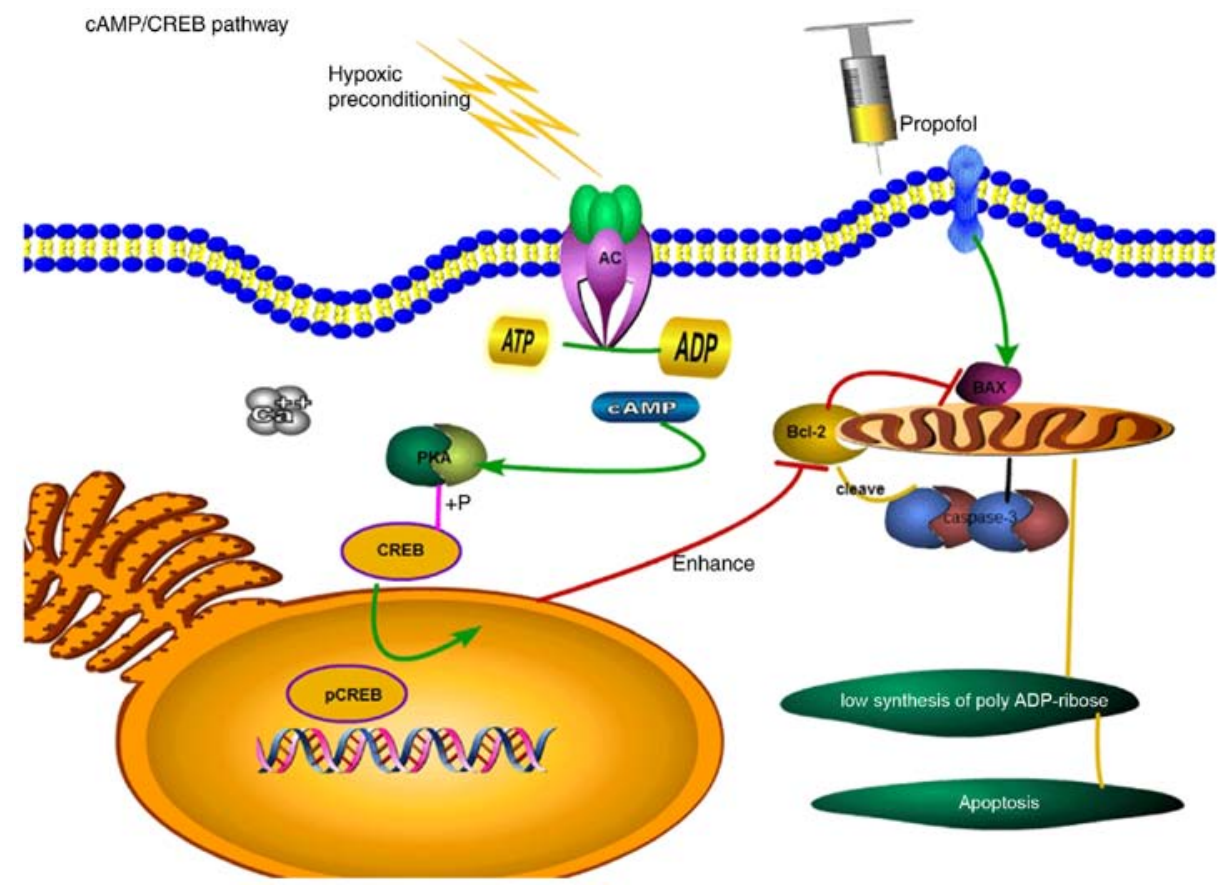

Figure 1. Role of the cAMP/CREB pathway in intrinsic apoptosis. cAMP, cyclic adenosine monophosphate; CREB, cAMP response element-binding protein.

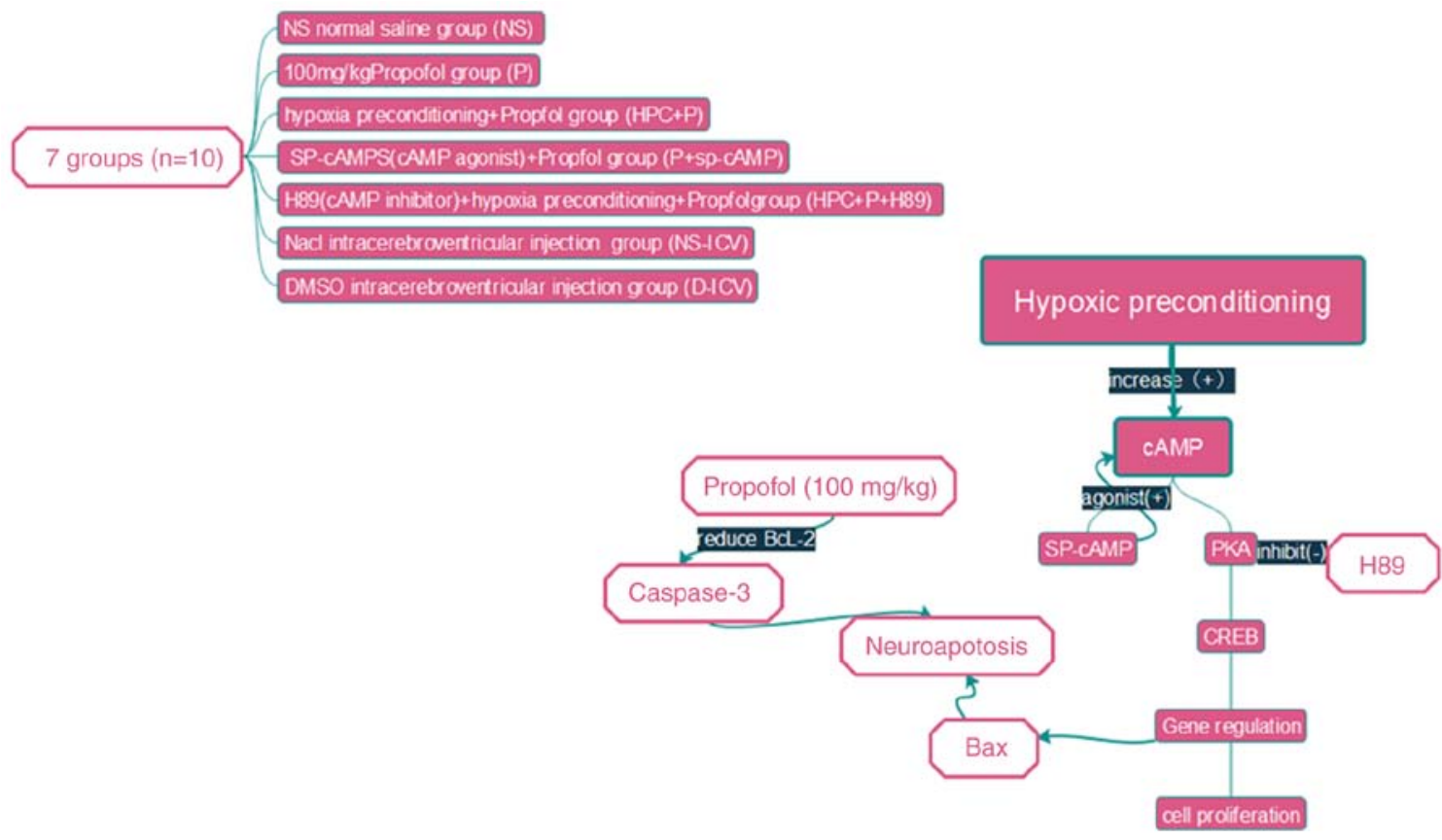

Figure 2. Experimental workflow.

were revealed to the investigator only after the completion of experiments and analyses. All pups were housed in a temperature-controlled room $\left(22 \pm 1^{\circ} \mathrm{C}\right)$ with a 12 -h light/dark schedule. H89 (Selleck Chemicals) and Sp-cAMP (Sigma-Aldrich; Merck KGaA) were prepared in $5 \mu 1$ double-distilled water. The experimental set-up is illustrated in Fig. $2(\mathrm{n}=10)$ and the following experimental groupings were used: i) Normal saline group (NS group) received intraperitoneal injections of an equal volume of normal saline; ii) propofol group (P group) received intraperitoneal injections of $100 \mathrm{mg} / \mathrm{kg}$ propofol; iii) following the propofol treatment as in the P group, the propofol + Sp-cAMP group (P+Sp-cAMP group) received intracerebroventricular injections of $20 \mathrm{nmol} / 5 \mu \mathrm{l} \mathrm{Sp}$-cAMP (a cAMP-dependent protein kinase agonist); iv) HPC+P group rats were placed in a chamber containing $8 \%$ oxygen and $92 \%$ nitrogen for $10 \mathrm{~min}$, and the pups were subsequently exposed to room air for a further $10 \mathrm{~min}$, and following five HPC cycles, the rats received an intraperitoneal injection of $100 \mathrm{mg} / \mathrm{kg}$ propofol; v) $\mathrm{HPC}+\mathrm{P}+\mathrm{H} 89$ group was exposed to $5 \mu \mathrm{mol} / 5 \mu \mathrm{l}$ H89 [a protein kinase A (PKA) inhibitor] by intracerebroventricular injections, followed by the same protocol as in the HPC+P group; vi) the remaining pups in the two blank test 
groups received intracerebroventricular injections of dimethyl sulfoxide (D-ICV group) or normal saline (NS-ICV group). All pups were sacrificed according to standard protocols (100 mg/kg intraperitoneal sodium pentobarbital). Brain tissue slices were prepared for immunohistochemistry and the levels of PKA, CREB, phosopho (p)-CREB, B-cell lymphoma 2 (Bcl-2), Bcl-2-associated $\mathrm{X}$ protein (Bax) and caspase-3 were evaluated by western blotting. Morphological and structural changes were evaluated by haematoxylin and eosin (H\&E) staining and transmission electron microscopy.

Intraventricular injections. As aforementioned, rats were anesthetized with sodium pentobarbital and centralized coordinates of anterior fontanel $(x=0, y=0, z=0)$ using stereotaxic apparatus (Ryward Life Technology Co., Ltd.), the sterile cannula was implanted at AP-2 mm (front and posterior), MLR-1.5 mm (left and right of the midline), and $\mathrm{H}-2 \mathrm{~mm}$ (depth from the left ventricle, $x=-1.0 \mathrm{~mm}, y=2 \mathrm{~mm}, \mathrm{z}=0$ ). After positioning, the skull was drilled, and then Sp-cAMP $(5 \mu \mathrm{l})$ or H89 $(5 \mu \mathrm{l})$ was slowly injected at rate of $0.1 \mu \mathrm{l} / \mathrm{min}$. The blank groups following the same protocol with an equal volume of DMSO or normal saline.

ELISA. The intracellular concentrations of adenylyl cyclase in the pups was determined by ELISA according to the instructions of the assay manufacturer (cat. no. S0026; Beyotime Institute of Biotechnology).

Western blot analysis. All pups were sacrificed to harvest the brain tissue. The protein was extracted by RIPA Lysis Buffer (Beijing Solarbio Science \& Technology Co., Ltd.) and protein concentration measured using a bicinchoninic acid protein assay (Biotype Biotech Co.). The mass of protein loaded per lane was $20 \mu \mathrm{l}$. Equal amounts of proteins were loaded onto $12 \%$ SDS-polyacrylamide gels. Electrophoresed proteins were transferred to polyvinylidene difluoride membranes $(0.22-\mu \mathrm{m}$ pore size; EMD Millipore). The membranes were blocked using 5\% bovine serum albumin (blocking buffer) for $2 \mathrm{~h}$ at room temperature and incubated with the following primary antibodies overnight at $4^{\circ} \mathrm{C}$ : $\beta$-tubulin $(1: 2,000$; cat. no. 48885$)$, caspase-3 (1:1,000; cat. no. 48658) and cleaved caspase-3 (1:1,000; cat. no. 29034; all from Signalway Antibody) Bcl-2 (1:1,000; cat no. ab196495; Abcam), Bax (cat. no. 27727) PKA (cat. no. 5842S), CREB (cat. no. 9197S) and p-CREB (cat. no. 9198S) (all 1:1,000; from Cell Signaling Technology, Inc.), and GAPDH (1:10,000; cat. no. 10494-1-AP; Proteintech, Inc.). The membranes were washed three times with Tris-buffered saline 1\% Tween-20 (TBST; pH 7.4) and then incubated in horseradish peroxidase-conjugated secondary antibody (1:10,000; cat. no. 134658; LI-COR Biosciences) for $2 \mathrm{~h}$ at room temperature $\left(23-25^{\circ} \mathrm{C}\right)$ and washed three times with TBST. The bands were developed using an Odyssey infrared imaging system (LI-COR Biosciences) and evaluated using densitometric analysis (ImageJ $1.52 \mathrm{~h}$, National Institutes of Health).

$H \& E$ and immunohistochemical staining. Morphological and structural changes were observed by H\&E staining. Tissues were fixed in $4 \%$ ice-cold paraformaldehyde at $4^{\circ} \mathrm{C}$ for $2 \mathrm{~h}$ and paraffin-embedded sections were obtained. The paraffin sections were dewaxed in xylene for $15 \mathrm{~min}$ and rehydrated using graded ethanol. The sections were immersed in haematoxylin for $30 \mathrm{sec}$ and then subjected to antigen retrieval using $0.01 \mathrm{~mol} / \mathrm{l}$ sodium citrate and incubated with $10 \%$ normal goat serum at room temperature for $30 \mathrm{~min}$ to block nonspecific binding, followed by incubation with the primary antibodies against PKA C and p-CREB (cat. nos. 5842S and 9198S, 1:1,000; Cell Signaling Technology, Inc.) at $4^{\circ} \mathrm{C}$ overnight. The sections were incubated with streptavidin-horseradish peroxidase at room temperature for $30 \mathrm{~min}$ and then stained with $0.05 \%$ 3,3-diaminobenzidine substrate, followed by counterstaining with $1 \%$ haematoxylin at $37^{\circ} \mathrm{C}$ for $30 \mathrm{sec}$. The sections were observed using a microscope (Olympus BX53; Olympus Corporation) and four fields of the hippocampus were randomly selected in every section which represented the areas of interest and the positive cells were counted using Image-Pro Plus version 6.0 software (Media Cybernetics Inc.).

Electron microscopy. The ultrastructures of neurocytes were observed by transmission electron microscopy (HITACHI H-7650; Hitachi, Ltd.). Briefly, 2.5\% glutaraldehyde solution was perfused into the rats, and the tissues were fixed in $1 \%$ $\mathrm{OsO}_{4}$ at $4^{\circ} \mathrm{C}$ for $1 \mathrm{~h}$, dehydrated in increasing concentrations of ethanol and embedded in Epon. Then, the samples were sectioned into semi-thin slices $(1 \mu \mathrm{m})$ and stained with $1 \%$ uranyl acetate and $5 \%$ uranyl acetate at $37^{\circ} \mathrm{C}$ for $20 \mathrm{~min}$. The ultrastructures of the entire mitochondria were measured by manually measuring length using Image Pro Plus (version 6.0.0.260, Media Cybernetics, Inc.).

Statistical analysis. Data are presented as the mean \pm standard error, and were analysed using SPSS version 17.0 (SPSS, Inc.) and GraphPad Prism 5 software (GraphPad Software Inc.). Multiple comparisons were performed using one-way analysis of variance (ANOVA), followed by Dunnett's post hoc test, as appropriate. $\mathrm{P}<0.05$ was considered to indicate a statistically significant difference.

\section{Results}

HPC induces CREB phosphorylation and attenuates propofol-induced neurotoxicity in neonatal rats. $\mathrm{H} \& \mathrm{E}$ staining revealed areas of brain cells affected by propofol, which exhibited less shrunken cell bodies and pyknotic nuclei (Fig. 3). Compared with the P group, electron microscopy demonstrated that neurons were rich in mitochondria and the width of mitochondrion was decreased in the $\mathrm{HPC}+\mathrm{P}$ group ( $\mathrm{F}=54.44, \mathrm{P}<0.05$; Fig. 4). Treatment with H89, a PKA inhibitor, revealed expansion of the width of mitochondrion similar to the $\mathrm{P}$ group.

$H P C$ increases the level of $C A M P$ and PKA, and p-CREB-positive cells. The level of cAMP was significantly upregulated in the $\mathrm{HPC}+\mathrm{P}$ group compared with the $\mathrm{P}$ group (Fig. 5). Additionally, western blot analysis revealed a significant difference in PKA levels between the P group and the NS group. The expression of Bcl-2 was significantly decreased in the pups treated with propofol; whereas, the expression of Bax and cleaved caspase-3 was significantly increased (Fig. 6A-D). Western blot analysis demonstrated that the level of p-CREB 

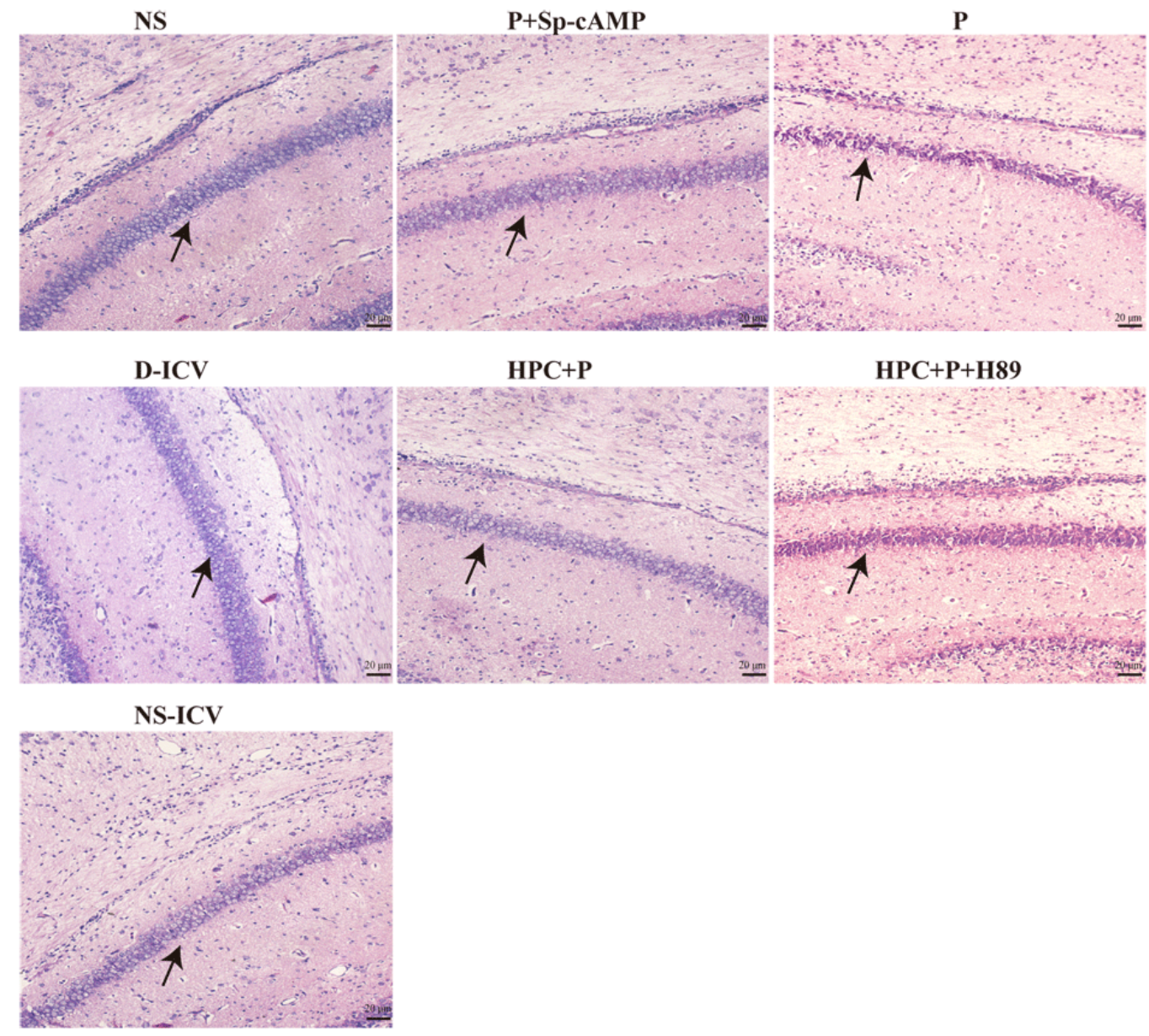

Figure 3. Haematoxylin and eosin staining of brain lesions. Examination by light microscopy following a high dose injection of propofol. Histological analysis revealed normal cells in the three blank test groups (NS, NS-ICV, and D-ICV) and disordered cells in the P group, which exhibited different degrees of degeneration. Furthermore, cellular degeneration in the HPC+P and P+Sp-cAMP groups was mild. Magnification, $\mathrm{x} 200$. Black arrow indicates the CA1 area of the Hippocampus. NS, normal saline; ICV, intracerebroventricular; D, dimethyl sulfoxide; P, propofol; HPC, hypoxic preconditioning; Sp-cAMP, cyclic adenosine monophosphate agonist.

was significantly increased in the HPC+P group compared with the P group $(1.050 \pm 0.083$ vs. $1.400 \pm 0.111$, respectively, $\mathrm{F}=15.83$; $\mathrm{P}<0.05$; Fig. $6 \mathrm{E}$ and $\mathrm{F}$ ). The results of immunohistochemical staining indicated that HPC and treatment with Sp-cAMP (a cAMP-dependent protein kinase agonist) increased the levels of PKA $(\mathrm{F}=17.26 ; \mathrm{P}<0.05 ;$ Fig. 7) and p-CREB ( $F=14.81 ; \mathrm{P}<0.05$; Fig. 8), whereas H89 abolished these effects.

Propofol reduces the number of $p$-CREB-positive cells in the hippocampal CAl region. The expression of p-CREB was negatively associated with the expression of cleaved-caspase-3, and there was no significant difference in the expression of CREB between the NS and NS-DIV groups (Fig. 8).

HPC suppresses propofol-induced neurotoxicity via activation of cAMP-dependent proteins. Compared with the NS group, the activities of cleaved caspase-3 were upregulated with a 2-fold change in the $\mathrm{P}$ group, and the levels of Bax were examined to investigate neurotoxicity following treatment with $100 \mathrm{mg} / \mathrm{kg}$ propofol. In the HPC+P group, the Bcl-2 (Fig. 6) and p-CREB (Fig. 8) levels in brain tissue were significantly increased compared with the $\mathrm{P}$ group, which was consistent with the Sp-cAMP treatment. Furthermore, H89 decreased the activation of p-CREB following HPC compared with the HPC+P group (Fig. 8).

\section{Discussion}

The present findings are an extension of previous studies $(18,19)$ to investigate the cAMP/PKA/CREB signalling pathway and propofol-induced apoptosis. The results of the present study revealed that p-CREB promotes cell survival and long-term synaptic change. Propofol-induced intrinsic apoptosis in neonatal rats was previously reported to be mediated by Bax (20) and release of caspase-3, which are important hallmarks of apoptosis (21). Bax disrupts mitochondrial membrane potential by affecting the permeability transition pores and facilitating the release of cytochrome $c$ (22).

PKA expression was reduced in the $\mathrm{P}$ group and this reduction was prevented by HPC, but not when H89 was applied. Furthermore, cAMP levels were markedly increased in the HPC $+\mathrm{P}$ group. These data confirmed that HPC attenuates propofol-induced neuroapoptosis by altering the content of cAMP in the hippocampus of rats via activation of cAMP/PKA/CREB signalling, a reduced $\mathrm{Bax} / \mathrm{Bcl}-2$ ratio and 
A

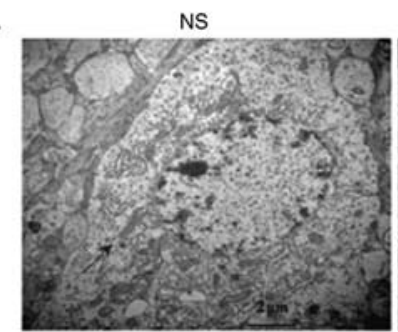

D-ICV

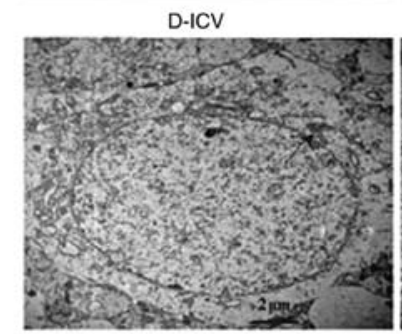

NS-ICV

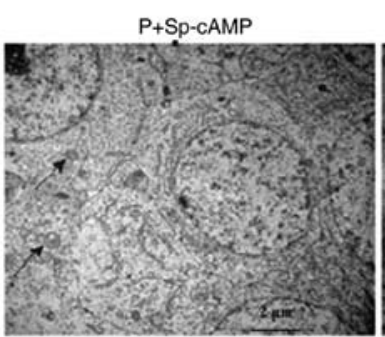

$\mathrm{HPC}+\mathrm{P}$

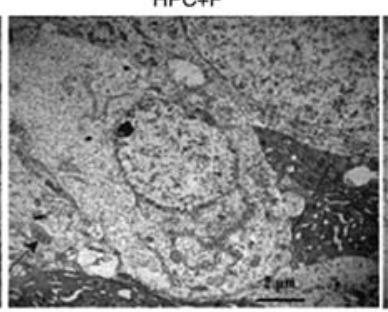

$P$

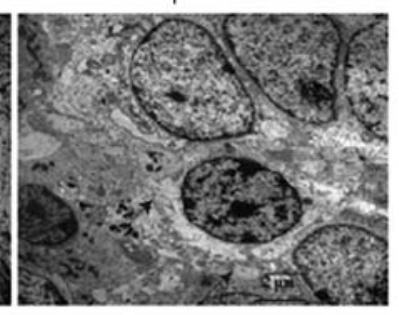

$\mathrm{HPC}+\mathrm{P}+\mathrm{H} 89$

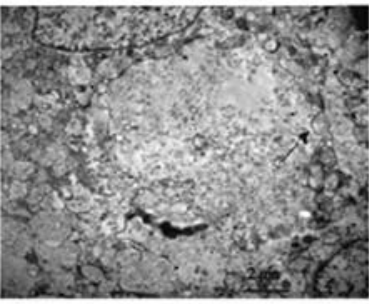

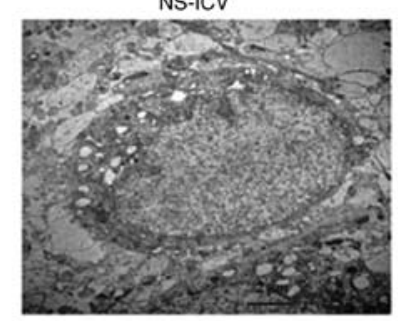

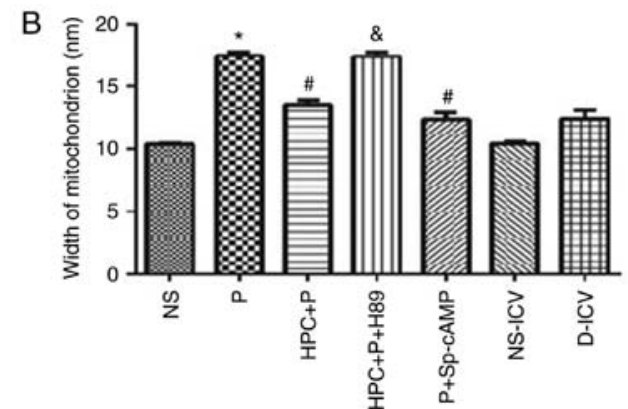

Figure 4. Examination of the hippocampal CA1 region by transmission electron microscopy (Magnification, x15,000) following propofol-induced apoptosis (A) Dendritic spine lesions, endoplasmic reticulum degranulated and mitochondrial swelling were observed. (B) Width of mitochondrion were measured by Image Pro Plus (version 6.0.0.260), $\mathrm{F}=54.44$; ${ }^{*} \mathrm{P}<0.05$ vs. the NS group; ${ }^{\text {}} \mathrm{P}<0.05$ vs. the $\mathrm{P}$ group; ${ }^{\text {}} \mathrm{P}<0.05$ vs. the HPC+P group. NS, normal saline; $\mathrm{P}$, propofol; HPC, hypoxic preconditioning; Sp-cAMP, cyclic adenosine monophosphate agonist; ICV, intracerebroventricular; D, dimethyl sulfoxide.

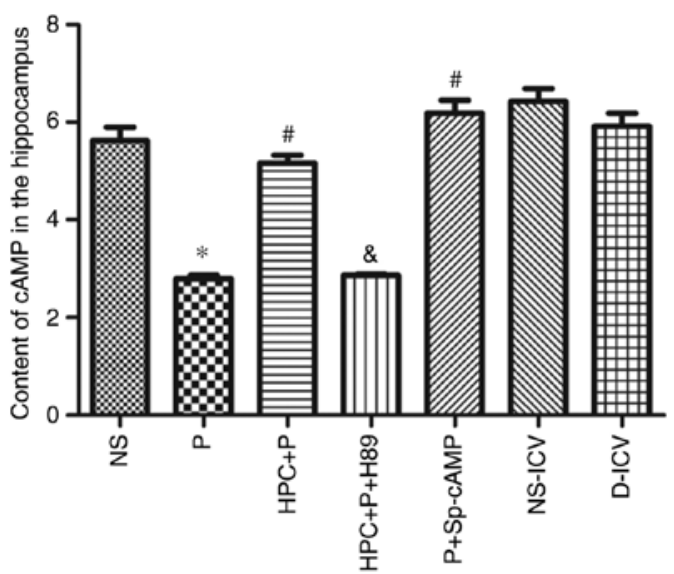

Figure 5. ELISA analysis of cAMP content in the hippocampus of rats. $\mathrm{F}=50.58,{ }^{*} \mathrm{P}<0.05$ vs. the NS group; ${ }^{\#} \mathrm{P}<0.05$ vs. the $\mathrm{P}$ group; ${ }^{\&} \mathrm{P}<0.05$ vs. the HPC+P group. cAMP, cyclic adenosine monophosphate; NS, normal saline; $\mathrm{P}$, propofol; HPC, hypoxic preconditioning; Sp-cAMP, cyclic adenosine monophosphate agonist; ICV, intracerebroventricular; D, dimethyl sulfoxide. downregulation of caspase-driven apoptosis downstream. The relative expression of these proteins determines cell survival. Caspase- 3 is one of the key executors of apoptosis and a widely studied member of the caspase family.

Mitochondrial outer membrane permeabilization isinvolved in the neuroprotective effects of HPC against propofol-induced neurotoxicity in rats. The study by Xu et al (23) revealed that HPC promotes the survival and viability of trophoblast cells. Transmission electron microscopy demonstrated that the mitochondrial outer membrane and matrix were enhanced by HPC with less degenerating vacuoles and apoptotic bodies observed. The findings indicated that pretreament with HPC reduced mitochondrial apoptosis.

Neuronal cell apoptosis is associated with minor behavioural changes and cognitive dysfunction in adolescent rats (24). When an apoptosis signal appears, cleaved caspase- 3 causes degradation of the neuron cell membrane and prevents the repair of damaged DNA. HPC induces robust 


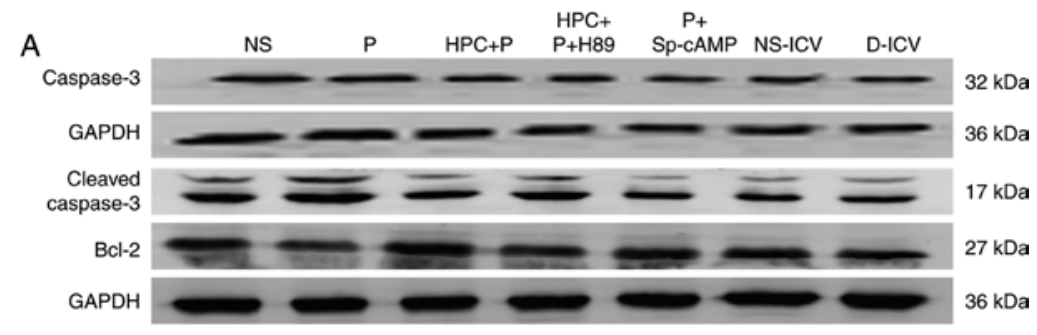

B
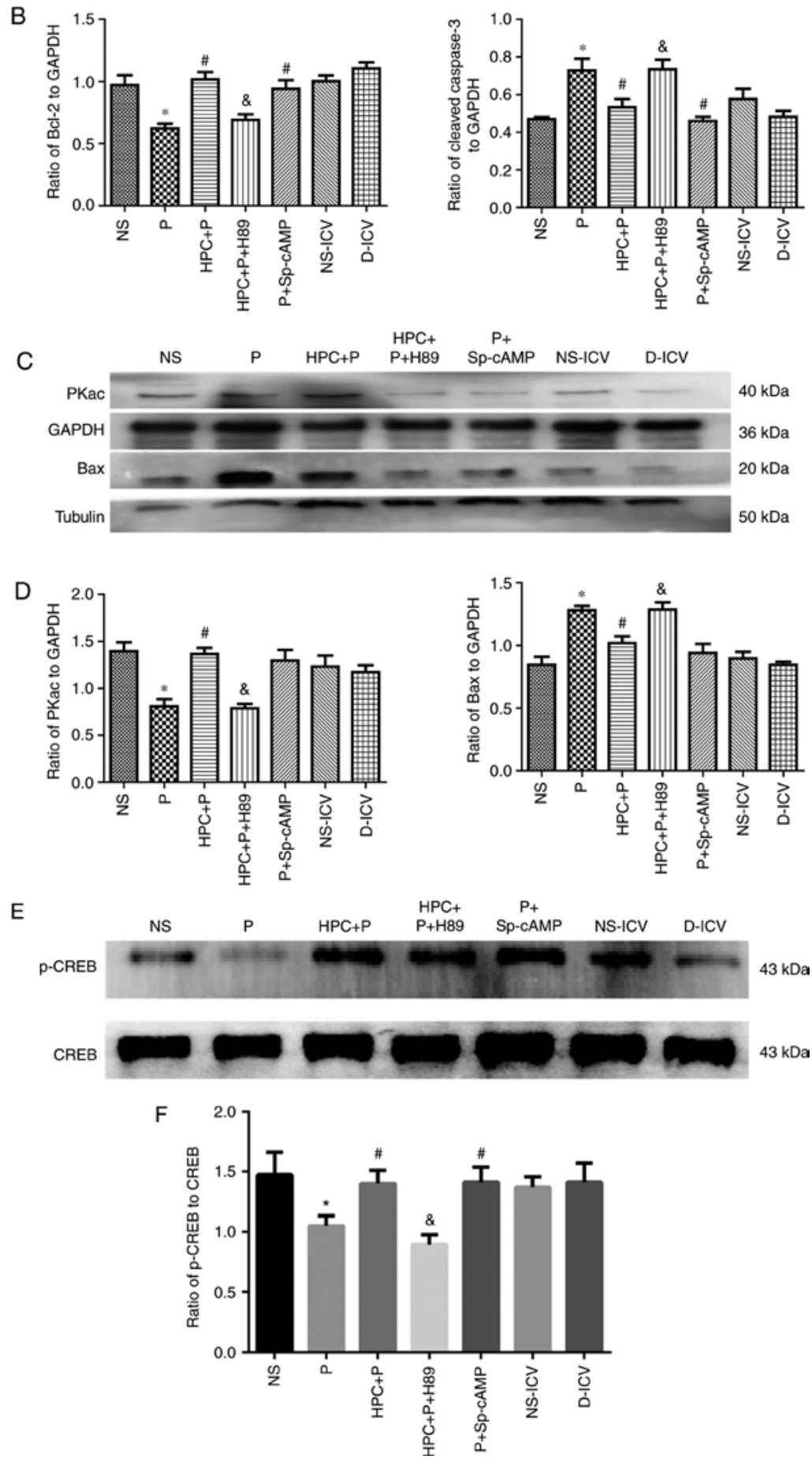

Figure 6. Activation of cAMP/PKA/CREB by HPC or cAMP agonists reduces neuroapotosis in the developing brains of Sprague-Dawley rats ( $\mathrm{n}=10$ per group). (A-D) Representative western blots using 12\% SDS-PAGE and densitometry analysis of the ratio of of Bax to $\beta$-tubulin ( $\mathrm{F}=12.81), \mathrm{Bcl}-2$ ( $\mathrm{F}=9.990)$, cleaved caspase-3 ( $\mathrm{F}=7.409)$, PKAc ( $\mathrm{F}=8.366)$, to GAPDH. Compared with the NS group, the expression of cleaved caspase-3 was increased to a 2 -fold change in the P group; neonatal exposure to HPC attenuated the effect of propofol-induced apoptosis with downregulation of cleaved caspase-3. (E and F) Representative western blots using $12 \%$ SDS-PAGE and densitometry analysis of the ratio of $\mathrm{p}$-CREB to CREB $(\mathrm{F}=15.83)$. The results are expressed as the mean \pm standard deviation. ${ }^{*} \mathrm{P}<0.05$ vs. the NS group; ${ }^{\text {}} \mathrm{P}<0.05$ vs. the $\mathrm{P}$ group; ${ }^{\circledR} \mathrm{P}<0.05$ vs. the HPC+P group. NS, normal saline; $\mathrm{P}$, propofol; HPC, hypoxic preconditioning; Sp-cAMP, cyclic adenosine monophosphate agonist; ICV, intracerebroventricular; D, dimethyl sulfoxide; cAMP, cyclic adenosine monophosphate; PKAc, protein kinase A catalytic subunit; CREB, cAMP response element-binding protein; Bcl-2, B-cell lymphoma-2; Bax, Bcl-2-associated X protein. 
A
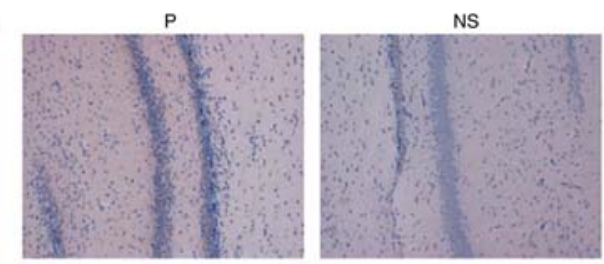

$\mathrm{HPC}+\mathrm{P}$

D-ICV
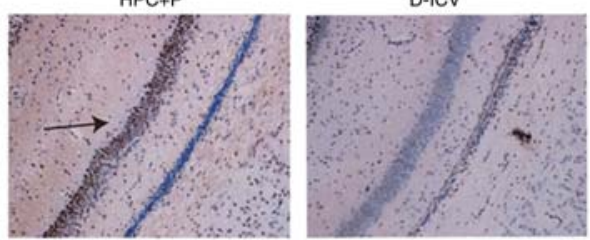

$\mathrm{HPC}+\mathrm{P}+\mathrm{H} 89$

NS-ICV
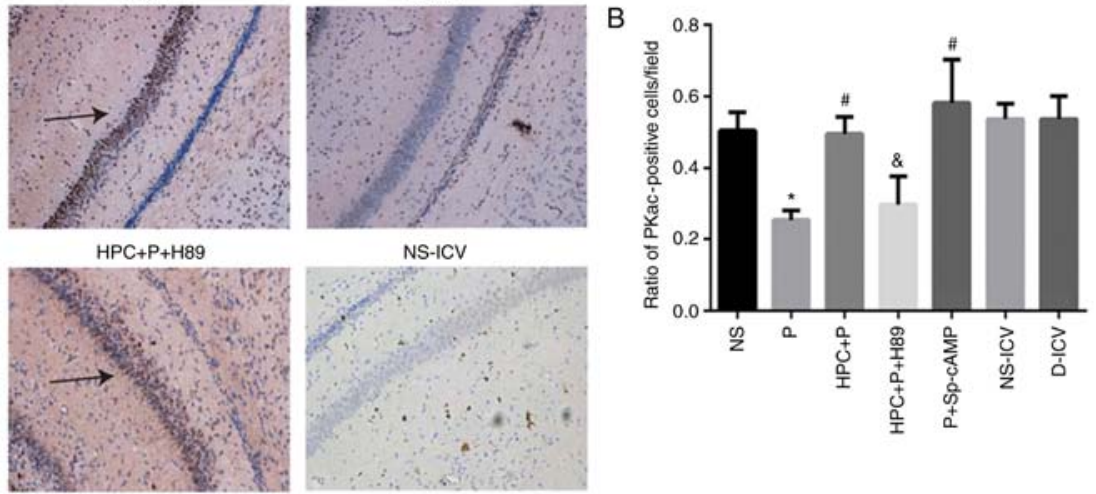

P + Sp-CAMP

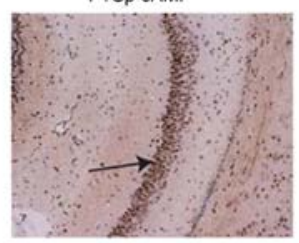

Figure 7. Effect of propofol on PKA expression following HPC. (A) Immunohistochemical staining of PKA in the seven groups (Magnification, x100). (B) Quantification of PKA staining expressed as the mean \pm standard deviation; $\mathrm{F}=17.26,{ }^{*} \mathrm{P}<0.05$ vs. the NS group; ${ }^{*} \mathrm{P}<0.05$ vs. the $\mathrm{P}$ group; ${ }^{\text {\& }} \mathrm{P}<0.05$ vs. the HPC+P group. Black arrow indicateS PKA-positive cells. PKA, protein kinase A; NS, normal saline; P, propofol; HPC, hypoxic preconditioning; Sp-cAMP, cyclic adenosine monophosphate agonist; ICV, intracerebroventricular; D, dimethyl sulfoxide.

A

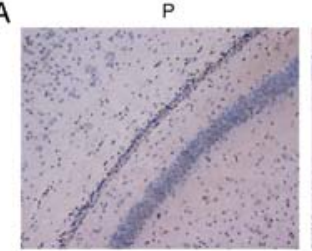

$\mathrm{HPC}+\mathrm{P}$

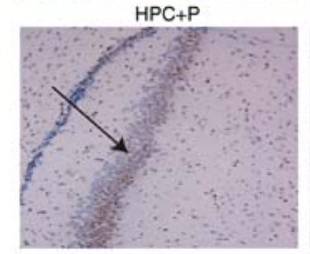

$\mathrm{HPC}+\mathrm{P}+\mathrm{H} 89$

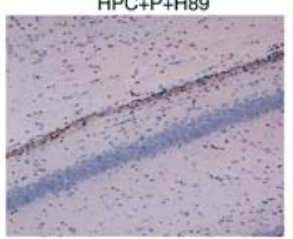

P+Sp-CAMP

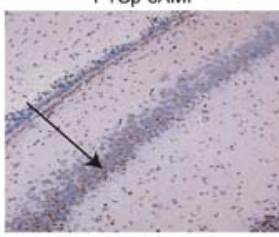

NS

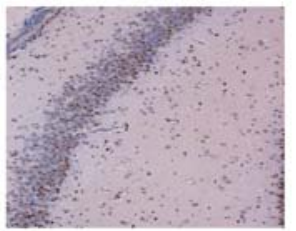

D-ICV

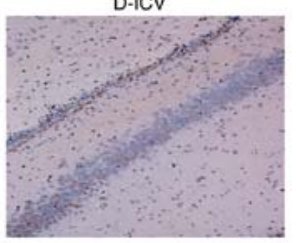

NS-ICV

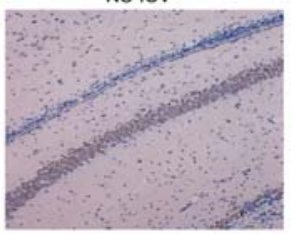

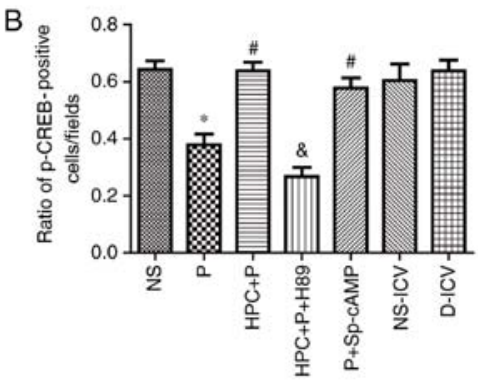

Figure 8. Effect of propofol on CREB following HPC. (A) Immunohistochemical staining of p-CREB in the seven groups (Magnification, $\mathrm{x} 100$ ). (B) Quantification of $\mathrm{p}-\mathrm{CREB}$ staining expressed as the mean \pm standard deviation; $\mathrm{F}=14.81,{ }^{*} \mathrm{P}<0.05$ vs. the NS group; ${ }^{\text {}} \mathrm{P}<0.05$ vs. the $\mathrm{P}$ group; ${ }^{\circledR} \mathrm{P}<0.05$ vs the $\mathrm{HPC}+\mathrm{P}$ group. Black arrow indicateS p-CREB positive cells. p, phospho; CREB, cAMP response element-binding protein; NS, normal saline; $\mathrm{P}$, propofol; HPC, hypoxic preconditioning; Sp-cAMP, cyclic adenosine monophosphate agonist; ICV, intracerebroventricular; D, dimethyl sulfoxide. 
neuroprotection in models of neonatal hypoxia (25). HPC was demonstrated to increase $\mathrm{Bcl}-2$ expression and reverse the propofol-induced reduction in neuronal cAMP levels. Additionally, as a potent and selective PKA inhibitor for evaluation of PKA function in many organs, such as the brain, muscle and heart, pretreatment with H89 (26), abolished the beneficial effects of HPC, whereas pretreatment with $\operatorname{Sp}$-cAMP $(27,28)$, a PKA agonist, increased the protective effects of HPC on propofol-induced hippocampal apoptosis. Whether Sp-cAMP exhibits a neuronal protective effect on the hippocampus requires investigation in future experiments, since our results do not include H89 or Sp-cAMP alone. Notably, cellular life and death are mitigated by Bcl-2 (29), which may be correlated with the cAMP/PKA/CREB pathway balancing the neuronal proliferation and apoptosis, as the phosphorylation of CREB promotes synaptic and neural plasticity, and $\mathrm{Bcl}-2$ mediates mitochondria-induced cellular toxicity (30).

The mechanism underlying the neuroprotective effect of HPC is associated with the cAMP content. The findings of the present study revealed that the apoptosis rate was significantly decreased in the $\mathrm{HPC}+\mathrm{P}$ and $\mathrm{P}+\mathrm{Sp}-\mathrm{cAMP}$ groups compared with the $\mathrm{P}$ group. Thus, HPC ameliorates propofol-induced neuroapoptosis via an increase in cAMP levels and phosphorylation of CREB, which prevents caspase-3 from inducing the apoptosis of hippocampal neurons.

\section{Acknowledgements}

Not applicable.

\section{Funding}

The present study was supported by the National Natural Science Foundation of China (grant nos. 81373498 and 81060277), the Guangxi Key Research and Development Program (grant no. AB18221031), the Science Study and Technology Development Program of Guangxi (grant no. 1355005-4-2), and the Science and Technology Research Project of Guangxi University (grant no. 2013ZD014).

\section{Availability of data and materials}

The datasets used during the present study are available from the corresponding author upon reasonable request.

\section{Authors' contributions}

YX was responsible for the conception and design of the study. YX, RG, JL, YT and FX conducted the experiments. LL analysed the data, RG drafted the work and revised it critically for important intellectual content. All authors have read and approved the final manuscript and agree to be accountable for all aspects of the research in ensuring that the accuracy or integrity of any part of the work are appropriately investigated and resolved.

\section{Ethics approval and consent to participate}

All animal procedures were conducted with the approval of the Animal Care and Use Committee of Guangxi Medical University (Nanning, China).

\section{Patient consent for publication}

Not applicable.

\section{Competing interests}

The authors declare that they have no competing interests.

\section{References}

1. Miner JR and Burton JH: Clinical practice advisory: Emergency department procedural sedation with propofol. Ann Emerg Med 50: 182-187, 187.e1, 2007.

2. Vasileiou I, Xanthos T, Koudouna E, Perrea D, Klonaris C, Katsargyris A and Papadimitriou L: Propofol: A review of its non-anaesthetic effects. Eur J Pharmacol 605: 1-8, 2009.

3. Wang J, Cottrell JE and Kass IS: Effects of desflurane and propofol on electrophysiological parameters during and recovery after hypoxia in rat hippocampal slice CA1 pyramidal cells. Neuroscience 160: 140-148, 2009.

4. Creeley C, Dikranian K, Dissen G, Martin L, Olney J and Brambrink A: Propofol-induced apoptosis of neurones and oligodendrocytes in fetal and neonatal rhesus macaque brain. $\mathrm{Br}$ J Anaesth 110 (Suppl 1): i29-i38, 2013.

5. Ko HM, Kim SY, Joo SH, Cheong JH, Yang SI, Shin CY and Koo BN: Synergistic activation of lipopolysaccharide-stimulated glial cells by propofol. Biochem Biophys Res Commun 438: 420-426, 2013

6. Sun WC and Pei L: rno-miR-665 targets BCL2L1 (Bcl-xl) and increases vulnerability to propofol in developing astrocytes. J Neurochem 138: 233-242, 2016.

7. Logan S, Jiang C, Yan Y, Inagaki Y, Arzua T and Bai X: Propofol alters long non-coding RNA profiles in the neonatal mouse hippocampus: Implication of novel mechanisms in anesthetic-induced developmental neurotoxicity. Cell Physiol Biochem 49: 2496-2510, 2018.

8. Heckman PRA, Blokland A, Bollen EPP and Prickaerts J: Phosphodiesterase inhibition and modulation of corticostriatal and hippocampal circuits: Clinical overview and translational considerations. Neurosci Biobehav Rev 87: 233-254, 2018.

9. Zhang S, Liang Z, Sun W and Pei L: Repeated propofol anesthesia induced downregulation of hippocampal miR-132 and learning and memory impairment of rats. Brain Res 1670: 156-164, 2017.

10. Kajimoto M, Atkinson DB, Ledee DR, Kayser EB, Morgan PG, Sedensky MM, Isern NG, Des Rosiers C and Portman MA: Propofol compared with isoflurane inhibits mitochondrial metabolism in immature swine cerebral cortex. J Cereb Blood Flow Metab 34: 514-521, 2014.

11. Cui Y, Ling-Shan G, Yi L, Xing-Qi W, Xue-Mei Z and Xiao-Xing Y: Repeated administration of propofol upregulated the expression of c-Fos and cleaved-caspase-3 proteins in the developing mouse brain. Indian J Pharmacol 43: 648-651, 2011.

12. Lv J, Liang Y, Tu Y, Chen J and Xie Y: Hypoxic preconditioning reduces propofol-induced neuroapoptosis via regulation of $\mathrm{Bcl}-2$ and Bax and downregulation of activated caspase- 3 in the hippocampus of neonatal rats. Neurol Res 40: 767-773, 2018.

13. Baillieul S, Chacaroun S, Doutreleau S, Detante O, Pépin JL and Verges S: Hypoxic conditioning and the central nervous system: A new therapeutic opportunity for brain and spinal cord injuries? Exp Biol Med (Maywood) 242: 1198-1206, 2017.

14. Okuda S, Sufu-Shimizu Y, Kato T, Fukuda M, Nishimura S, Oda T, Kobayashi S, Yamamoto T, Morimoto S and Yano M: CaMKII-mediated phosphorylation of RyR2 plays a crucial role in aberrant $\mathrm{Ca}^{2+}$ release as an arrhythmogenic substrate in cardiac troponin T-related familial hypertrophic cardiomyopathy. Biochem Biophys Res Commun 496: 1250-1256, 2018.

15. Liang C, Du F, Cang J and Xue Z: Pink1 attenuates propofol-induced apoptosis and oxidative stress in developing neurons. J Anesth 32: 62-69, 2018.

16. Wei ZZ, Zhu YB, Zhang JY, McCrary MR, Wang S, Zhang YB, Yu SP and Wei L: Priming of the cells: Hypoxic preconditioning for stem cell therapy. Chin Med J (Engl) 130: 2361-2374, 2017.

17. Tsui YP, Mung AK, Chan YS, Shum DK and Shea GK: Hypoxic preconditioning of marrow-derived progenitor cells as a source for the generation of mature schwann cells. J Vis Exp: Jun 14, 2017. doi: $10.3791 / 55794$. 
18. Lv J, Wei Y, Chen Y, Zhang X, Gong Z, Jiang Y, Gong Q, Zhou L, Wang $\mathrm{H}$ and Xie Y: Dexmedetomidine attenuates propofol-induce neuroapoptosis partly via the activation of the PI3k/Akt/GSK3 $\beta$ pathway in the hippocampus of neonatal rats. Environ Toxicol Pharmacol 52: 121-128, 2017.

19. Zhong Y, Liang Y, Chen J, Li L, Qin Y, Guan E, He D, Wei Y, Xie Y and Xiao Q: Propofol inhibits proliferation and induces neuroapoptosis of hippocampal neurons in vitro via downregulation of NF- $\kappa \mathrm{B}$ p 65 and $\mathrm{Bcl}-2$ and upregulation of caspase-3. Cell Biochem Funct 32: 720-729, 2014.

20. Galluzzi L and Vanpouille-Box C: BAX and BAK at the gates of innate immunity. Trends Cell Biol 28: 343-345, 2018.

21. Rahmani M, Nkwocha J, Hawkins E, Pei X, Parker RE, Kmieciak M, Leverson JD, Sampath D, Ferreira-Gonzalez A and Grant S: Cotargeting BCL-2 and PI3K induces BAX-dependent mitochondrial apoptosis in AML cells. Cancer Res 78: 3075-3086, 2018.

22. Ma ZW and Liu DX: Humanin decreases mitochondrial membrane permeability by inhibiting the membrane association and oligomerization of Bax and Bid proteins. Acta Pharmacol Sin 39: 1012-1021, 2018.

23. Xu C, Li X, Guo P and Wang J: Hypoxia-induced activation of JAK/STAT3 signaling pathway promotes trophoblast cell viability and angiogenesis in preeclampsia. Med Sci Monit 23: 4909-4917, 2017.

24. Karen T, Schlager GW, Bendix I, Sifringer M, Herrmann R, Pantazis C, Enot D, Keller M, Kerner T and Felderhoff-Mueser U: Effect of propofol in the immature rat brain on short- and long-term neurodevelopmental outcome. PLoS One 8: e64480, 2013.
25. Stetler RA, Leak RK, Gan Y, Li P, Zhang F, Hu X, Jing Z, Chen J, Zigmond MJ and Gao Y: Preconditioning provides neuroprotection in models of CNS disease: Paradigms and clinical significance. Prog Neurobiol 114: 58-83, 2014.

26. Bao D, Zhao W, Dai C, Wan H and Cao Y: H89 dihydrochloride hydrate and calphostin $\mathrm{C}$ lower the body temperature through TRPV1. Mol Med Rep 17: 1599-1608, 2018.

27. Yoo SB, Lee S, Lee JY, Kim BT, Lee JH and Jahng JW: cAMP/PKA agonist restores the fasting-induced down-regulation of nNOS expression in the paraventricular nucleus. Korean J Physiol Pharmacol 16: 333-337, 2012.

28. Laycock JF, Hubbard JI, Schwartz JH, Stanton BA and Valtin H: The cAMP agonist Sp-cAMPS stimulates osmotic water transport across rat inner medullary collecting duct cells. Ann N Y Acad Sci 689: 606-608, 1993.

29. Singh R, Letai A and Sarosiek K: Regulation of apoptosis in health and disease: The balancing act of BCL-2 family proteins. Nat Rev Mol Cell Biol 20: 175-193, 2019.

30. Lonze BE and Ginty DD: Function and regulation of CREB family transcription factors in the nervous system. Neuron 35 : 605-623, 2002.

(i) $(9)$ This work is licensed under a Creative Commons Attribution-NonCommercial-NoDerivatives 4.0 International (CC BY-NC-ND 4.0) License. 